\title{
Measurements of Electroencephalogram (EEG), Galvanic Skin Resistance (GSR) and Heart Rate Variability (HRV) during the Application of a System that Gives Simultaneously tVNS and Brain Entrainment on Subjects Affected by Depression and Anxiety
}

\author{
Conte $\mathrm{S}^{1}$, Casciaro $\mathrm{F}^{1,7}$, Wang $\mathrm{F}^{\mathbf{8}}$, Altamura \\ $M^{1,2}$, Bellomo A ${ }^{2}$, Serafini $G^{1,3}$, Orsucci F , \\ Kaleagasioglu $F^{1,5}$, Mendolicchio $L^{1,6}$, Norman $R^{1}$ \\ and Conte $\mathrm{E}^{1 *}$ \\ ${ }^{1}$ School of Advanced International Studies on Applied \\ Theoretical and non Linear Methodologies of Physics, \\ Italy \\ ${ }^{2}$ Department of Biomedical Science, University of Foggia, \\ Italy \\ ${ }^{3}$ Tor Vergata University, Italy \\ ${ }^{4}$ Division of Psychology, University College, England \\ ${ }^{5}$ Department of Pharmacology, Faculty of Medicine, Near \\ East University, Mersin 10, Turkey \\ ${ }^{6}$ Clinic Villa Miralago, ASL Varese, Italy \\ ${ }^{7}$ Department of Biomedical Sciences and Human \\ Oncology, University of Bari, Italy \\ ${ }^{8}$ College of Science/ Agricultural Mathematical Modeling \\ and Data Processing Center, Human Agricultural \\ University, China \\ *Corresponding author: Conte E, School of Advanced \\ International Studies on Applied Theoretical and non \\ Linear Methodologies of Physics, Italy
}

Received: August 02, 2018; Accepted: September 04, 2018; Published: September 11, 2018

\section{Introduction}

The device that we have used, is a system realized by the NuCalm (http://www.nucalm.com/NuCalm-Clinical/how-nucalm-works. $\mathrm{cfm}$ ) designed with the purpose to treat subjects affected by depression, anxiety, stress. The whole system consists of four components that leverage biochemistry, neurophysiology, and physics to obtain and maintain parasympathetic nervous system dominance.

Previously, we have performed an HRV analysis just on tVNS only [1-4].

Let us express how the four components work together. First, NuCalm topical cream is applied to the carotid on the neck and it is designed to act counteracting adrenaline. It includes structured, nutrient-sourced building blocks (Active ingredients are PharmaGABA, Lactium (Casein Tryptic Hydroylsate), L-Theanine and L-Tyrosine) that rapidly create a natural relaxation response. Second, the system uses the micro current stimulation patches, a tVNS system that is placed behind each ear at the Mastoid Process. Research has shown that this device induces an increase of parasympathetic response [1]. Next, a noise- dampening headphones are used to deliver proprietary nonlinear oscillating neuroacoustic software to bring the brain wave pace to the pre-sleep stages $(12 \mathrm{~Hz}$ $-4 \mathrm{~Hz}$ ) via brain entrainment. Finally, Light-blocking eye masks are used to negate visual stimuli.

The aim of the present paper is to study the combination of these four components in subjects having Psychological Disorders (P.D.) as depression and anxiety. We have studied the Electroencephalogram (EEG), the Galvanic Skin Resistance (GSR) level and the HRV during the complete treatment in subjects affected by anxiety and/ or depression.

\section{Materials and Methods}

Solace Lifesciences, Inc., of Wilmington, Delaware, USA, furnished the NuCalm device. 100 young subjects affected by anxiety, or depression were examined.

To test the subjects each potential participant was given a nonstructured interview following the criteria of the Diagnostic and Statistical Manual of Mental Disorders (DSM-V). Participants selected for the study showed symptomatology of anxiety or depression.
Ann Depress Anxiety - Volume 5 Issue 2 - 2018 ISSN : 2381-8883 | www.austinpublishing group.com Conte et al. (C) All rights are reserved 


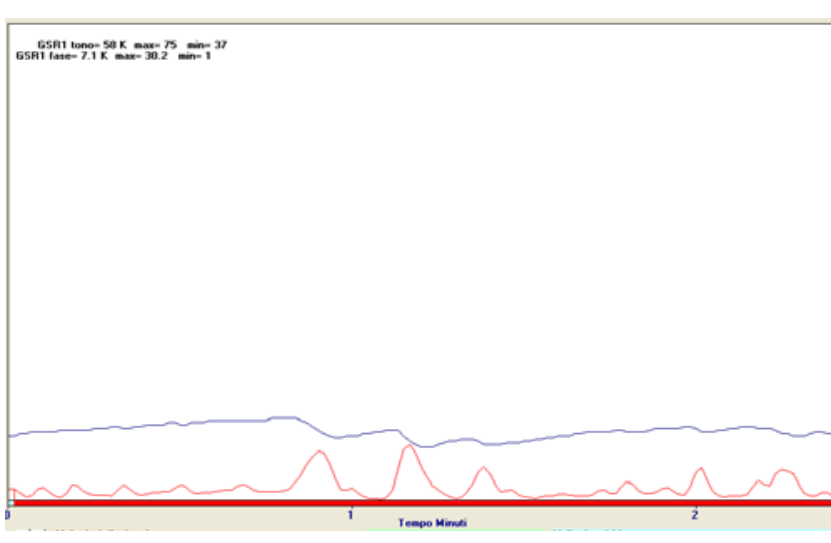

Figure 1: Improvements during the NuCalm treatment.

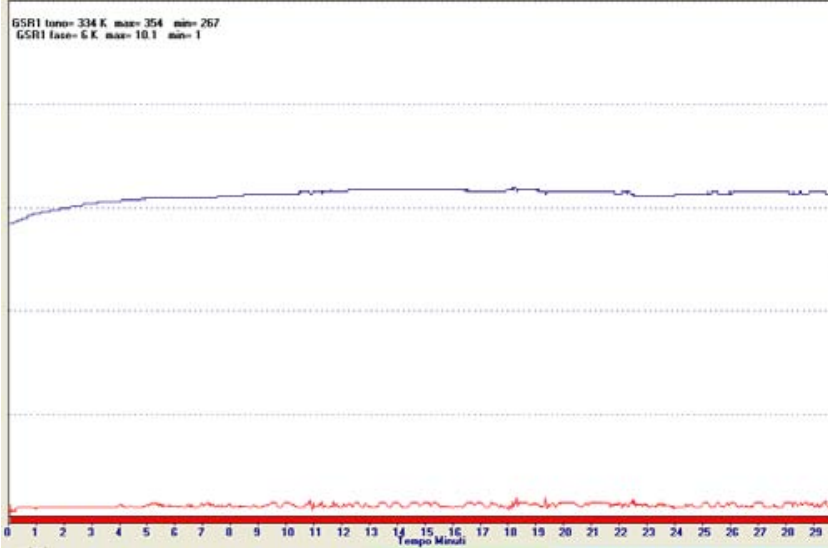

Figure 2: GSR during the treatment.

Those selected were divided into two groups and administered tests to evaluate the level of their anxiety and depression symptoms. The scales used to measure their scores were, respectively, the Hamilton Anxiety Scale (HAM-A) and the Hamilton Depression Scale (HAM-D). Both tests were administered during patient recruitment and showed medium scores, with HAM-A at 41 and HAM-D at 19.

Specifically, we examined all between the ages of 25 and 45 . All the subjects gave their written informed consent. According to the 1996 Task Force Standards of the European Society of Cardiology and the North American Society of Pacing and Electrophysiology, selected subjects had only one or more of the two-targeted psychiatric pathologies, and did not have high blood pressure, cardio circulatory insufficiency, diabetes, or chronic diseases. In particular, subjects who had heart-related disease, Coronary Artery Disease (CAD), heart failure, or irregular heartbeat were excluded. We excluded subjects who had abnormal Q wave, WPW syndrome, hypertrophy of the left ventricle or cardiac hypertrophy. Subjects with an R-R interval below $90 \%$ of the normal range were also excluded.

For one hour prior to the exam, no drinking or food was allowed. Ear clip Plethysmography (PG) was performed at 9:30 a.m., with subjects in a comfortable sitting position, with soft and distant indoor light, and with appropriate indoor temperatures $\left(20^{\circ} \mathrm{C}-23^{\circ} \mathrm{C}\right.$, $\left.68^{\circ} \mathrm{F}-74^{\circ} \mathrm{F}\right)$. The same conditions were used for the Galvanic Skin

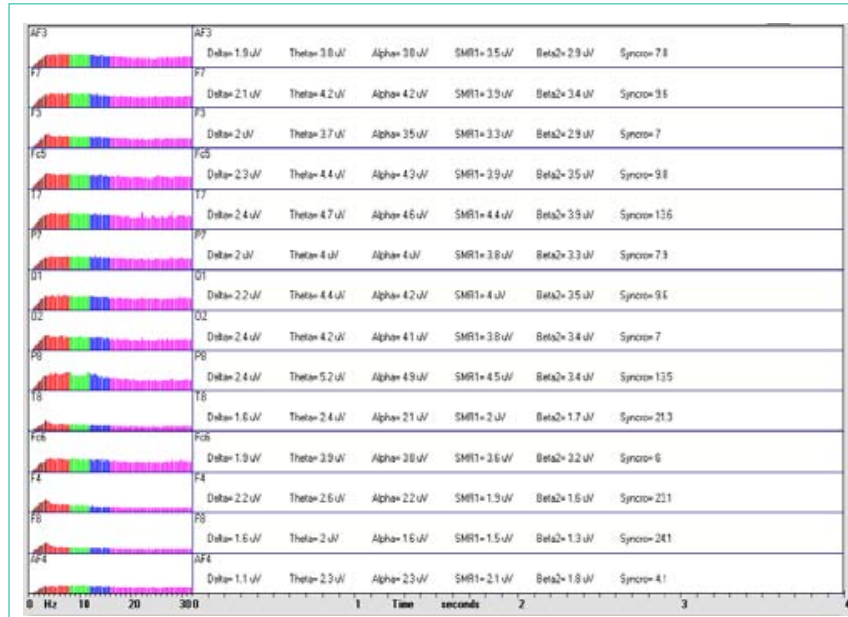

Figure 3: Let us examine the situation during the NuCalm treatment.

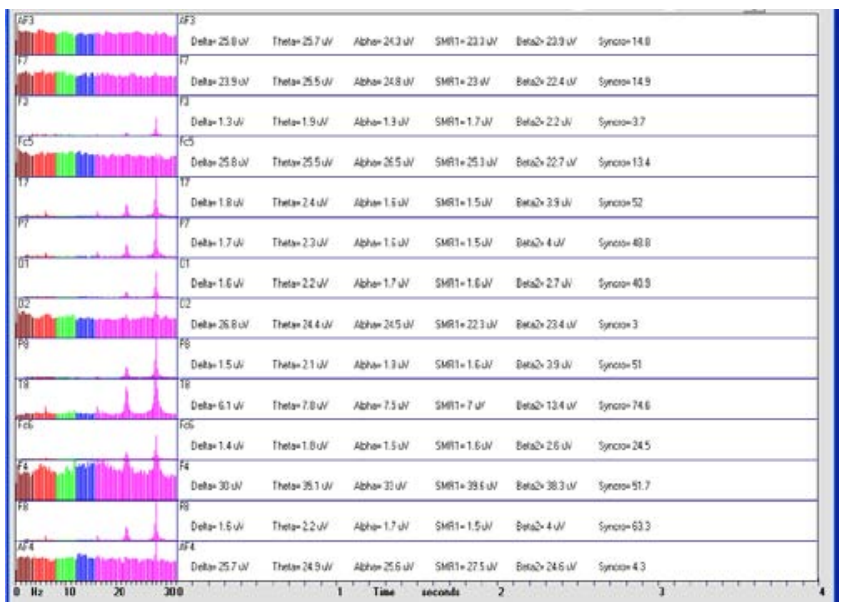

Figure 4: The intra hemispherical as well as inter hemispherical synchronization are reported.

Response (GSR) and for the EEG. A preparatory recording period of at least three minutes was given for the cream administration and then the exam was conducted under the tVNS and Brain Entrainment administration. The obtained tachograms recorded by PG were compared to those we obtained by ECG, accounting for Pulse Wave Transit Time (PWTT).

The NuCalm tVNS was applied with gel electrode patches near the right and left ear lobes (Mastoid Process), and setting the current value at $0.1 \mathrm{~mA}$. The treatment was given for 30 minutes. The measurement of the GSR was performed by two electrodes of silver attached to the phalanxes of the fingers, index and annular, of the hand. The EEG was performed by using a wireless device and a complete poligraphy consisting of HR, GSR and EEG signals were obtained.

\section{Results}

\section{Let us start with the results obtained by the GSR}

First, we measured the GSR values before the treatment. Considering that the normal value of the tone is between 100 and 700 $\mathrm{kOhm}$, a mean value of $61 \pm 23 \mathrm{kOhm}$ for the 100 subjects indicated the pathological stressed state of the subjects. The phases indicate a 


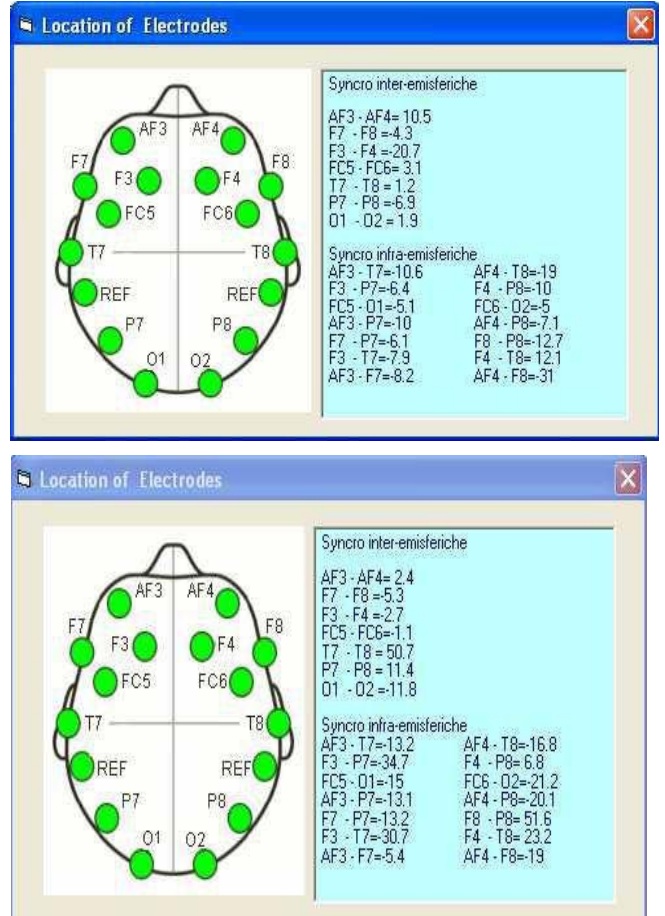

Figure 5: Location of Electrodes.

rather conservative system with spontaneous and continuous activity of the peak value with normal half time recovery. Overall we have a pathological status of depression and anxiety. Here is an example: we report the case of a subject showing a very restricted mean value to $58 \mathrm{kOhm}$ with a minimum value of $37 \mathrm{kOhm}$ and a maximum of $75 \mathrm{kOhm}$ for the tone. The phase indicates a turbulent condition of pathology since we observe a continuous activity determined from the unconscious state that induces different peaks at so restricted time intervals (Figure 1)

\section{Let us see the improvements during the NuCalm treatment}

The mean value of the tone rises to $420 \pm 39 \mathrm{k} \mathrm{Ohm}$. In the example case of the above subject, we have a rather constant behavior of the tone $(334 \mathrm{k} \mathrm{Ohm})$ with a maximum value of 354 and a minimum of $267 \mathrm{k} \mathrm{Ohm}$. We outline the total absence of peaks. In addition, notice the very fast induction of the relaxation response signed from absence of peaks on the phase (Figure 2).

\section{Let us investigate the EEG}

Let us examine the case of the electroencephalogram by qEEG inspecting the behavior for bands. For the delta we have values that are in the interval between 1.00 and 2.70 microVolts. For the Theta bands, we obtained values that range between 2.00, 5.00 microvolts, and Alpha band ranging from 1.30 to 5.00 micro Volts. In the figure, we report the example case of the same subject before explored by the GSR. We observe that all the examined area is activated but the alpha values are very low as well as is low the value of the synchronization (Figure 3).

\section{Let us examine the situation during the NuCalm treatment}

We see that, apart from the F3, F7, O1 and P6 area, all the other areas are stimulated from the treatment and in particular are

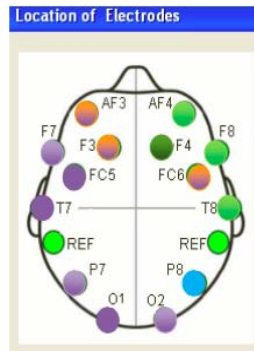

Before the treatment - Thet

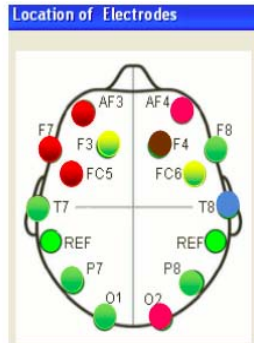

During the treatment - Theta

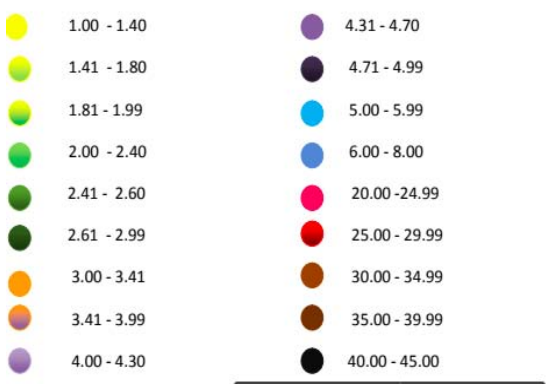

Figure 6: Location of Electrodes - Theta treatment.

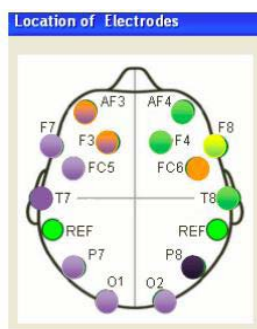

Before the treatment - Alpha

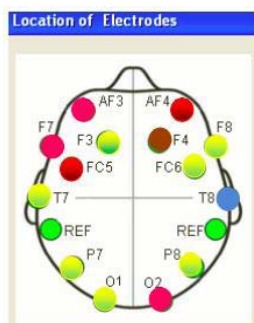

During the treatment - Alpha

\begin{tabular}{|c|c|}
\hline $1.00-1.40$ & $4.31-4.70$ \\
\hline $1.41-1.80$ & $4.71-4.99$ \\
\hline $1.81-1.99$ & $5.00-5.99$ \\
\hline $2.00-2.40$ & $6.00-8.00$ \\
\hline $2.41-2.60$ & $20.00-24.99$ \\
\hline $2.61-2.99$ & $25.00-29.99$ \\
\hline $3.00-3.41$ & $30.00-34.99$ \\
\hline $3.41-3.99$ & $35.00-39.99$ \\
\hline $4.00-4.30$ & $40.00-45.00$ \\
\hline
\end{tabular}

Figure 7: Location of Electrodes - Alpha treatment.

stimulated the values of the delta, theta, and alpha bands respect to the case of no stimulation. The values of synchronization are increased. The mean value of the 100 subjects for delta is $22.00 \pm 5.00$ microvolts, the mean value of the theta is $21.00 \pm 6.00$ microvolts and the mean value of the alpha are $25 \pm 7.00$ microvolts. In Figure below we report the values of the example subject (Figures 4-9).

We now consider the HRV. In absence of the treatment the 


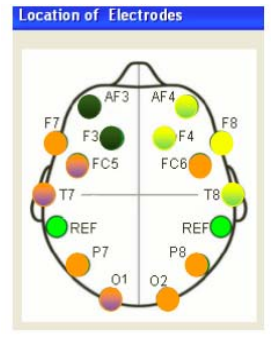

Before the treatment - Beta2

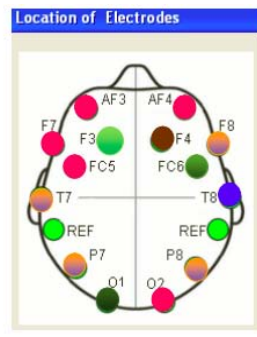

During the treatment - Beta2

\begin{tabular}{|c|c|}
\hline $1.00-1.40$ & $4.71-4.99$ \\
\hline $1.41-1.80$ & $5.00-5.99$ \\
\hline $1.81-1.99$ & $6.00-8.00$ \\
\hline $2.00-2.40$ & $8.01-13.50$ \\
\hline $2.41-2.60$ & $20.00-24.99$ \\
\hline $2.61-2.99$ & $25.00-29.99$ \\
\hline $3.00-3.40$ & $30.00-34.99$ \\
\hline $3.41-3.99$ & $35.00-39.99$ \\
\hline $4.00-4.30$ & $40.00-45.00$ \\
\hline & \\
\hline
\end{tabular}

Figure 8: Location of Electrodes - Beta2 treatment.

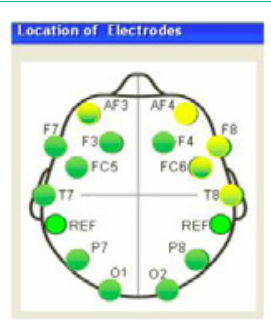

Before the treatment - Delta

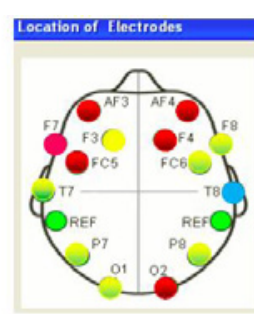

During the treatment - Delta

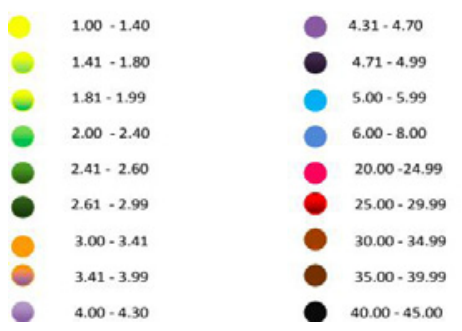

Figure 9: Location of Electrodes - Delta treatment.

situation is as follows. This is the tachogram of the above patient and it is followed from the FFT analysis of the Frequency Domain (Figure $10)$.

We see that the LF reaches to $1653 \mathrm{msec}^{2} / \mathrm{Hz}$ while the HF component is modulating action for this example stopped at 351 $\mathrm{msec}^{2}$. The ratio $\mathrm{LF} / \mathrm{HF}$ is equal to 4.71 . The mean value for the 100 subjects for the LF is $1100.00 \pm 500.00 \mathrm{msec}^{2}$. The mean value of the $\mathrm{HF}$ component is $\mathrm{HF}=400 \pm 200.00 \mathrm{msec}^{2}$.

During the treatment, we have the following value for the subject

The mean values were $299.00 \pm 102.00$ for LF and $300.00 \pm 100.00$

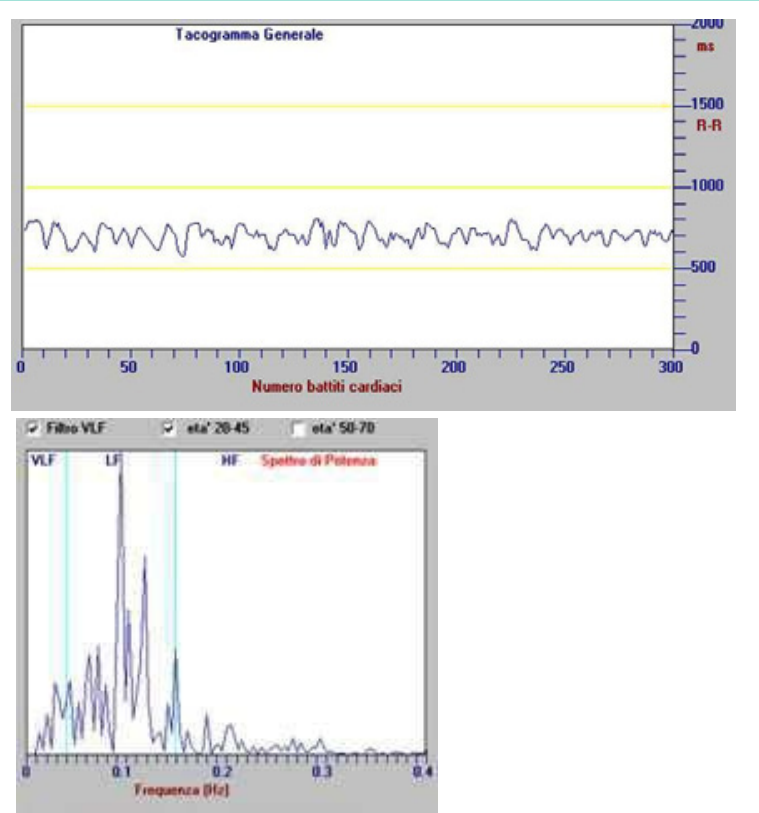

Figure 10: We see that the LF modulating action.
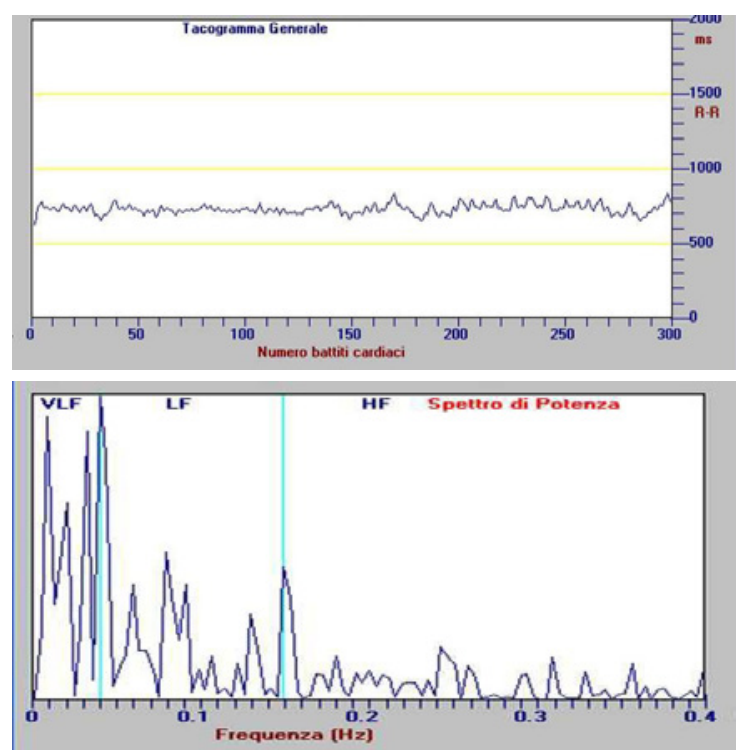

Figure 11: The results in during the treatment.

$\operatorname{msec}^{2}$ for HF. LF/HF = 0.99 (Figure 11).

\section{Conclusion}

We have studied the response of the brain system of depressed and anxious subjects to a combined stimulus that is made of supplement Cream + tVNS + Brain Entrainment and light blocking in the range $4-12 \mathrm{~Hz}$. We measured the response of the system by executing a complete poligraphy consisting of HR, GSR and qEEG. For the GSR we have evidenced that it shifted from a tone value of $61 \pm 23 \mathrm{kOhm}$ before of the treatment, indicating the pathological state of the subjects, to the normal value of $420 \pm 39 \mathrm{kOhm}$ during the treatment. A constant phase activity before the treatment disappeared 
during the treatment.

For qEEG we have obtained a great increase of delta, theta and alpha values after the treatment against a modest value of alpha activity before of the treatment. A high value of synchronization at intra and extra hemispheric indicates that the brain received a high benefit during the treatment.

For the HRV, we have obtained that the LF/HF value passed from 4.71 before of the treatment to 0.99 during the treatment. This result confirms that the NuCalm device operates at the level of increase of parasympathetic activity during the treatment. We conclude that the NuCalm is an optimum device able to produce a dominant parasympathetic state mixed to a drastic reduction of sympathetic activity.

\section{References}

1. Conte S, Wang F, Sala N, Casciaro F, Orsucci F, Serafini G, et al. The Transcutaneous Vagus Nerve Stimulation Output of the NuCalm Device of
Solace Lifesciences Is Found to Be a Multifractal and Therefore It Is Indicated in the Treatment of Heart Rate Variability in the Dysfunction of Autonomic Nervous System in Anxiety, Depression and Stress. Journal of Behavioral and Brain Sciences. 2017; 7.

2. Conte S, Wang F, Sala N, Casciaro F, Orsucci F, Serafini G, et al. Multifractal Analysis of Heart Rate Variability in Subjects Affected from Dysfunction of Autonomic Nervous System in Anxiety and Depression and Treated by Transcutaneous Vagus Nerve Stimulation. Austin Cardiology. 2018

3. Conte S, Sala N, Wang F, Serafini G, Kaleagasioglu F, Mendolicchio L, et al. The Transcutaneous Vagus Nerve Stimulation Device of the Nu-Calm-Solace Lifesciences Gives an Output Signal that is a Multifractal: Is there a Support in the Treatment of the Atrial Fibrillation? Austin Cardiology. 2017; 2: 1013.

4. Conte S, Wang F, Altamura M, Bellomo A, Serafini G, Orsucci F, et al. Analysis of Heart Rate variability (HRV) in Frequency Domain and Recurrence Quantification Analysis during the Treatment by Transcutaneous Vagus Nerve Stimulation. Ann Depress Anxiety. 2018.
Ann Depress Anxiety - Volume 5 Issue 2 - 2018

ISSN : 2381-8883 | www.austinpublishinggroup.com

Conte et al. (C) All rights are reserved
Citation: Conte S, Casciaro F, Wang F, Altamura M, Bellomo A, Serafini G, et al. Measurements of Electroencephalogram (EEG), Galvanic Skin Resistance (GSR) and Heart Rate Variability (HRV) during the Application of a System that Gives Simultaneously tVNS and Brain Entrainment on Subjects Affected by Depression and Anxiety. Ann Depress Anxiety. 2018; 5(2): 1095. 